\title{
Tangence
}

\section{L'héroïsme de la marge}

\section{Les essais de François Paré}

\section{François Ouellet}

Numéro 56, décembre 1997

Postures scripturaires dans la littérature franco-ontarienne

URI : https://id.erudit.org/iderudit/025957ar

DOI : https://doi.org/10.7202/025957ar

Aller au sommaire du numéro

Éditeur(s)

Tangence

ISSN

0226-9554 (imprimé)

1710-0305 (numérique)

Découvrir la revue

Citer cet article

Ouellet, F. (1997). L'héroïsme de la marge : les essais de François Paré.

Tangence, (56), 40-65. https://doi.org/10.7202/025957ar d'utilisation que vous pouvez consulter en ligne.

https://apropos.erudit.org/fr/usagers/politique-dutilisation/ 


\section{L'héroïsme de la marge. Les essais de François Paré François Ouellet}

En 1992, François Paré publiait Les littératures de l'exiguité, un essai dans lequel l'auteur soutient une réflexion dense sur les littératures dites marginales ${ }^{1}$ dans leur rapport à l'Institution littéraire, qui lui valut le prix du Gouverneur général du Canada en 1993. La littérature franco-ontarienne, qui y tenait une place signifiante, allait ensuite constituer l'essentiel du corpus d'étude de Théories de la fragilité, paru en 1994. Certainement, les études de Paré comptent pour beaucoup dans l'intérêt que l'on porte actuellement à la littérature franco-ontarienne, même s'il est vrai, comme il le dit lui-même, que - la conjoncture est très favorable aux marginalités ${ }^{2}$ et que *la qualité et [...] l'envergure de la production littéraire franco-ontarienne ${ }^{3}$ récente sollicitent le discours critique. Qu'il y ait eu une certaine reconnaissance du discours minoritaire dans la distinction institutionnelle des Littératures de l'exiguité ${ }^{4}$, cela fait peu de doute.

Pourtant, ce qui y apparaît particulier, c'est peut-être moins le corpus minoritaire dont il est question que le discours par lequel lessayiste saisit son objet, s'engageant résolument dans l'aventure de l'écriture. On sait que, parallèlement à la pratique théorique et à la recherche qui marquent les années soixante-dix et le début des années quatre-vingt, il y a eu la manifestation constante d'une critique littéraire soucieuse de son propre parcours, dans le sillage de la critique des Starobinski, Richard et Poulet. Je pense en particulier aux positions d'André Brochu, et plus encore à celles de Gilles Marcotte, qui rappelait récemment: "Je dois dire

1 L'auteur entend par là les littératures minoritaires, les littératures coloniales, les littératures insulaires et les petites littératures nationales.

2 François Paré, Les littératures de l'exiguitté, Ottawa, Le Nordir, 1992, p. 156. Dorénavant, j'indiquerai la pagination entre parenthèses après la citation.

3 François Paré, "Pour rompre le discours fondateur: la littérature et la détresse", dans Lucie Hotte et François Ouellet (dir.), La littérature franco-ontarienne: enjeux estbétiques, Ottawa, Le Nordir, 1996, p. 11.

4 Outre le prix du Gouverneur général, l'ouvrage a obtenu le Signet d'or 1993 de Radio-Québec. 
que les seuls critiques qui m'intéressent vraiment sont ceux qui sont des écrivains, qui participent à l'aventure de l'écriture. ${ }^{5} \mathrm{Et}$ s'il est vrai que les années quatre-vingt-dix font état de la fécondité de la recherche universitaire ${ }^{6}$, il apparaît toutefois qu'il s'y manifeste une critique singulièrement préoccupée d'une rencontre avec l'Autre plutôt que de l'imposition d'un regard dit scientifique sur le texte, une critique animée par la visée esthétique de son écriture et un savoir empirique plutôt que par l'élaboration d'une grille d'analyse et un savoir technique. Bref, comme le souligne Robert Dion, * [ill semble bien en effet que, dans la critique québécoise contemporaine, la ligne de fracture passe entre savoir et écriture, c'est-à-dire entre recherche et démarche "essayiste". Et, sauf à refuser d'exister sur la scène universitaire, c'est par rapport à ces pôles contraires que le critique doit désormais se situer ${ }^{7}$.

Dans cette veine de l'essai littéraire volontiers subjectif, c'està-dire marqué par une subjectivité qui agit comme "point de fuite de l'œuvre" ${ }^{8}$, les essais de François Paré occupent une place à part. Cela est en partie dû au fait que Paré radicalise remarquablement cette attitude essayiste, à la fois par sa position critique contre l'Institution hégémonique et par sa posture d'écrivain, adaptant à l'essai un mode discursif de type fictionnel, se faisant "un artiste de la narrativité des idées.9, selon la belle formule d'André Belleau. Position critique et posture d'écrivain sont du reste étroitement liées, car précisément la défiance de Paré à l'égard de l'Institution fait déjà le pari de modalités de production d'un discours qui s'écartera des canons esthétiques dominants. En somme, la richesse des essais ne tiendrait pas tant aux conditions socioculturelles franco-ontariennes qui auréolent l'ouvre étudiée, qu'à l'effet de discours produit, dont dépend directement la caution de l'auteur à la question posée et débattue. Il y a chez Paré

5 Pierre Popovic, Entretiens avec Gilles Marcotte. De la littérature avant toute chose, Montréal, Liber, 1996, p. 111. Il dit encore: "Quand j'écris un article ou une étude critique, cela prend souvent l'allure d'un récit . (p. 10).

6 On consultera par exemple Claude Duchet et Stéphane Vachon (dir.), La recherche universitaire. Objets et métbodes, Montréal, XYZ éditeur, 1993.

7 Robert Dion, . Critique universitaire et critique d'écrivain. Le cas d'André Brochu , Études littéraires, vol. 25, nos 1-2, été-automne 1992, p. 193-194.

8 André Brochu, - Critique et recherche *, La vtsée critique, Montréal, Boréal, 1988, p. 145.

9 André Belleau, *Petite essayistique., Surprendre les voix, Montréal, Boréal, 1986 , p. 86. 
une saisissante mise en récit du discours de la marge, une incomparable mise en scène littéraire de l'essayiste par lui-même, une puissante rhétorique de la sollicitation performative, affective, séduisante, perverse (le terme n'est nullement péjoratif: c'est la plus belle qualité que puisse se donner une écriture qui inscrit au sein de la linéarité du discours sa propre singularité), qui joue habilement de l'énonciation et des conditions socioculturelles dont elle témoigne. Je voudrais montrer, essentiellement dans Les littératures de l'exiguité, comment précisément l'essayiste-écrivain, parlant des textes des autres dans un contexte culturel minoritaire, parvient à l'écriture, au sens fort du terme, situant ses propres conditions d'écriture au cœur même du processus critique triomphant. Je m'intéresserai d'abord à l'inscription du *je *, du "nous" et du *il. dans l'essai, pour donner une vue d'ensemble de la dialectique fictionnelle, insistant en particulier sur sa structure victimaire et sacrificatoire et, à cet égard, sur la figure emblématique d'André Paiement. Enfin, la question du Père m'a paru opportune pour revisiter et clore l'ensemble du propos.

\section{La marque du « je »}

Dès l'incipit des Littératures de l'exiguité, Paré affirme éloquemment la position souveraine du moi auctorial vis-à-vis de l'objet, dont la capacité de transmission cognitive sera conditionnelle à l'investissement exemplaire qu'il subit.

J'écris ce livre face à la mer. C'est la mer du Nord, violente, inépuisable, imprévisible, au large de Katwijk aan Zee aux Pays-Bas. Voilà un paysage que je pourrais passer mes journées entières à recenser. Sur la plage, quelques vieux fouillent de leurs détecteurs de métal chaque centimètre carré de sable. Lorsque la mer est plus calme, des pêcheurs râtellent l'écume avec leurs filets souillés à la recherche de crevettes. Et, beaucoup plus loin, sans doute tordue par le vent, mais je n'en sais rien, brille parfois dans les rares éclaircies la plate-forme de forage, amenuisée par la distance (p. 5).

Dans une mise en scène hautement littéraire qui convoque le sérieux et le ludisme, qui se conditionnent mutuellement, l'essayiste se situe devant le paysage marin comme devant un texte à recenser. Avant d'être introduit sur un mode informatif, le "programme "de l'essai est défini par la métaphore. Ambitieux, curieux, en tout cas infatigable, l'essayiste sera sensible aux volontés discrètes, aux significations secrètes, enfouies dans 
l'étendue uniforme du langage, raclera même l'onde mouvementée, et, comme dans un éclair de génie - mais ici sa voix peut se perdre, la signification, fugitive, seulement s'entrevoir -, allant plus loin encore, creusera dans les sombres profondeurs encore vierges d'abordage, imprégnant sa propre pensée au texte à sonder, ici et là, manifestant les traces d'une quête (incertitude, tâtonnement, approche oblique) qu'il sait néanmoins * inépuisable "et "imprévisible. Le " je * manifeste sa trace exemplaire, s'affirme comme conscience subjective forte, - non comme garant de la vérité de son écrit mais comme conscience et mesure de sa portée. ${ }^{10}$.

Tour à tour, selon les besoins, la fouille des détecteurs, le filet jeté à la mer, la plate-forme de forage : nous avons là un condensé métaphorique de la démarche critique de Paré, tout en mouvements, jamais tout à fait fixe, se ménageant la surprise de l'exploration. Il écrit encore: "Lire les écriteaux, plantés ici et là, au gré des lectures, et puis me perdre tout de même: je suis cet homme. C'est pourquoi je sème au long de ce livre des textes témoins: découpures microscopiques des diverses marginalités. (p. 12). Paré affectionne la formule qui traduit la disponibilité à la découverte. Il notera : "Je suis tombé, comme par hasard et comme par enchantement, sur Tchicaya U Tam'si (le Congolais). (p. 49), ou encore: - le pays de Paul Savoie, où je me retrouve tout à coup. (p. 102), filant la métaphore initiale. L'attitude critique favorise une structure discursive hasardeuse, qui fait que le discours sera pleinement ouvert à l'inattendu. Dans cet ordre d'idées, Paré balise sa démarche critique, non pas en fonction des écrivains ou des textes, mais selon un découpage thématique des plus subjectifs, entremêlant des questions théoriques et critiques à des considérations socio-culturelles ou plus personnelles, avec une prédilection pour des titres atypiques, vagues et accrocheurs; témoin cette suite de titres: "La mouche des champs "; "Le spectacle ritualisé de la disqualification ", "L' "exiluité " ; "Le corps frêle de Florentine Lacasse *; "Parce que... *; *Une vertigineuse déshistoire"; "Le fou est entré dans notre maison "; "Le pays"; etc. Robert Vigneault a certainement raison quand il affirme que la table des matières de l'essayiste est souvent *imprévisibl[e] * et "déroutant[e]", mais je ne crois pas qu'il faille dire pour autant

10 Marc Angenot, La parole pampblétaire. Typologie des discours modernes, Paris, Payot, 1982, p. 57. 
qu'il a des * problèmes * avec sa table ${ }^{11}$. Les titres participent aussi de la littérature, qui ne cède jamais à la fonction didactique ${ }^{12}$. Paré privilégie ainsi un trajet qui mime l'aléatoire ${ }^{13}$, citant bon nombre d'écrivains qui passent et reviennent au fil de l'écriture, mouvement critique d'une pensée que Jean Marcel appelle *lyrique , car le discours *est fondé sur l'élément formel que constitue le retour rythmique des thèmes et des formulations" ${ }^{1 /}$. Or, si cette démarche s'explique par l'approfondissement visé de l'objet culturel, c'est avant tout parce qu'elle se définit par la pensée même de l'essayiste qui, plutôt que de prétendre à une explication logique, fait de sa propre interrogation, concomitante à l'objet culturel, le lieu privilégié d'où celui-ci peut être posé et validé, affirmé et relancé. Certes, "le texte de l'essai va plutôt se construire circulairement ou autour d'une pensée. ${ }^{15}$, mais d'abord parce que la pensée elle-même se pose comme enjeu d'une question. Nombre d'interrogations ou d'incertitudes traduisent, chez Paré, la quête, la recherche, la tentative de "la capture éphémère du sens. (p. 102) : : Pourquoi ce poème de Sylvain Garneau s'est-il imposé à moi? Est-ce parce que cet écrivain, mort très jeune comme André Paiement, suturait une brèche qui venait de s'ouvrir en moi [...]?. (p. 119). Il précisera : -Je fraie des voies. Du moins, c'est mon intention, mon travail. (p. 145). On ne saurait trouver plus belles traces d'une recherche intérieure, où l'écriture de la recherche cède à la recherche de l'écriture; où la part d'invention précède le développement, car c'est en se ménageant une marge de manœuvre pour la découverte que l'essayiste prendra la mesure exacte de son projet, parviendra à comprendre ce qu'il veut dire, ou mieux, ce qu'il peut dire, tant sa disponibilité

11 Robert Vigneault, L'écriture de l'essat, Montréal, l'Hexagone, 1994, p. 23-24.

12 Remarquable à cet effet la section intitulée - Fracasser les carreaux de la majson du Père •, ou Paré renvoie *à la lecture obligatoire-de l'essai de Patricia Smart, Écrire dans la maison du père, plutôt que de le commenter de quelque façon que ce soit.

13 Qui -mime*, c'est-à-dire qui n'exclut pas un certain agencement de l'aléatoire, un certain travail ludique de l'agencement. Le passage d'une section à l'autre se fait quelquefois selon un glissement thématique évident (ainsi une suite de sections sur la question de l'oralité), parfois selon un rapport plus ténu, qu'autorisent les rapprochements sémantiques de la thématique de l'exiguité: conscience humiliée, dépossession de l'Être, aliénation collective, etc.

14 Jean Marcel, *Prolégomènes à une théorie de l'essai •, Pensées, passions et proses, Montréal, l'Hexagone, 1992, p. 317.

15 Robert Vigneault, L'écriture de l'essai, op. cit., p. 29. 
s'investit autant vis-à-vis d'une relative facilité à cerner l'objet que vis-à-vis de sa profonde complexité: "Le domaine qui m'intéresse aujourd'hui est décidément très complexe, très difficile à saisir dans son ensemble. Je ne m'en doutais pas au départ *, avoue-t-il d'emblée (p. 9). "Au départ", car c'est précisément dans le trajet de la pensée que la question s'est développée.

Parce qu'il fraie dans l'inépuisable, dans ce qui ne se laisse jamais systématiser, l'essayiste admettra travailler dans l'absence de définition. "Mes problèmes de définition sont ici encore énormes. Est-ce pour cela que je me sers si souvent du mot " discours" ", dans la mesure où cette clé, empruntée pour l'occasion, m'ouvre toutes les portes?* (p. 48). Mais qu'importe, puisque l'indéterminable fait partie intégrante de la forme de l'essai, qui par définition n'épuise pas son sujet; ce qui n'empêche pas l'essayiste de désirer tout dire: "J'aurais voulu, si j'en avais eu la capacité linguistique, que ce livre soit universel [...]. Mais la connaissance des littératures dépasse les aptitudes du seul homme que je suis. [...] La liste est vertigineuse et excède, je l'avoue tout de suite, ma compréhension. (p. 7). Ainsi Paré se montre-t-il (volontiers) plus d'une fois dépassé par son sujet, faute de pouvoir se dépasser lui-même dans son projet. Mais ne redonne-t-il pas ainsi tout son sens au mot *essai *, où il s'agit d'éprouver, de supputer, d'essayer précisément, ou plutôt: de s'essayer, comme l'entendait Montaigne? Hugo Friedrich a mis en évidence la signification du mot dans la France de la Renaissance, et conséquemment de l'usage du genre lui-même, qui est * tout ce qui caractérise la pensée de Montaigne: le motif occasionnel, l'allure de la promenade, la conscience de l'insuffisance, la résignation de "se tenir à la rive" sans regret s'il ne peut avancer, mais aussi l'espoir qu'une pénétration plus profonde n'est pas interdite, - et au total le penchant à se trouver bien de toute cette passivité tâtonnante. ${ }^{10}$. Comment, à la lecture de ces lignes qui synthétisent si bien la sinueuse exemplarité du *je • chez Paré, ne pas songer que celui-ci est d'abord un spécialiste de la Renaissance, et que, au demeurant, il ait pu se souvenir, en écrivant l'incipit des Littérature de l'exiguité, du passage fameux des Essais auquel Friedrich fait ici allusion: "Si c'est un subject que je n'entende point, à cela mesme je l'essaye, sondant le gué de bien loing; et puis, le trouvant trop profond pour ma taille, je me tiens à la 
rive : et cette reconnoissance de ne pouvoir passer outre, c'est un traict de son effect, voire de ceux dequoy il se vante le plus " ${ }^{17}$ ? Chez Paré, plus que chez Starobinski, c'est Montaigne retrouvé.

Cela dit, s'il y a beaucoup d'improvisation dans le mouvement d'écriture de Paré, il ne faut pas pour autant s'y méprendre: il s'agit toujours d'une certaine mise en scène, d'un type de rapport à l'écriture. La démarche créative de l'essayiste, le plaisir de l'invention qui s'y rattache, masque l'ordre, qu'il soit rationnel ou profondément intuitif, dont dépend tout de même la lisibilité du discours: nous allons improviser ensemble, semble-t-il dire, mais vous me suivez. Ou en d'autres mots, comme le dit François Paré : "Le hasard des voyages a bien fait les choses. Celui des lectures dispersées aura fait le reste, je l'espère• (p. 8).

\section{La marque du « nous »}

Paré ponctue allègrement son texte de considérations qui soulignent la présence effective du moi à son discours, imposant sa présence par delà la marque linguistique: ainsi rappelle-t-il son travail au comité de rédaction du magazine franco-ontarien Liaison, son enseignement à l'Université de Guelph, des souvenirs d'une conférence de Lyotard * dans un vieux bâtiment de la Place de l'Odéon à Paris. (p. 58), ses réflexions à la sortie d'un film de Denys Arcand rue Sainte-Catherine, etc., toutes notations narratives ${ }^{18}$ qui créent une complicité indéniable avec le destinataire, renforcent l'intimité recherchée, ajoutent de la confidence au discours savant. L'essai est cousu d'intrusions d'auteur, comme on dit à propos de la fiction, celles-ci auront d'autant plus d'impact que le réel, au lieu d'être médiatisé comme dans l'œuvre de fiction, "n'existe que comme expérience ", la relation au vécu immédiat s'imposant comme "l'attitude essentielle de l'essayiste " ${ }^{19}$. Aussi toutes ces confidences d'auteur s'alimentent-elles à celle-ci, centrale, dont le coefficient d'authenticité qui s'y rattache est renforcé par une rhétorique de l'évidence, de l'insistance, de la connivence: "Je vais dire un truisme, mais je crois qu'il faut

17 Montaigne, Les essais, t. 1, Paris, Presses universitaires de France, 1988, p. 301 .

18 Comme dans l'incipit, nous retrouvons constamment dans l'essai le souci du décor, ce qui contribue au développement de séquences narratives.

19 Robert Vigneault, L'écriture de l'essat, op. cit., p. 22 et 40. 
le dire, pour que les choses soient claires entre nous: il n'est pas facile d'écrire et de vivre dans l'insularité et l'ambiguité d'une culture minoritaire et largement infériorisée. (p. 7). Le • je•, recenseur "en mouvement " de paysages divers, situe son discours dans le cadre d'un espace et d'une culture donnés, dont il participe, et vis-à-vis de la question fondamentale de l'identité; et, dans la mesure où elle est valable pour l'ensemble des littératures marginales, l'assertion a valeur universelle: c'est pourquoi la littérature franco-ontarienne paraîtra *si représentative de toutes les autres * (p. 80), témoignant d'une façon qui lui est propre, mais exemplaire, de la conscience de la marge.

Ainsi, l'écriture, ouvertement performative et chargée d'un̂' fort potentiel affectif, cherche à établir une forte complicité avec le lecteur dans le partage d'un point de vue. Si nous sommes loin ici de la forme pamphlétaire ou du discours pragmatique, il est clair que le lyrisme de Paré contient une visée perlocutoire insistante, use de toute une rhétorique allocutive fort habile, dont témoigne par exemple un *notre avenir collectif * (p. 18), qui présuppose l'adhésion du lecteur au discours et qui ambitionne éventuellement de le faire agir. Or, par son engagement vis-à-vis du lecteur, le discours performatif tend à reproduire la forme même de la relation de l'essayiste à la société marginale: il s'agit toujours de valoriser la forme "communale. Paré n'écrit pas à propos de ou sur, mais avec la littérature marginale. * Il arrive [...] que l'intérêt pour la littérature nationale soit moins l'intérêt que l'on porte à la chose écrite que la satisfaction déguisée de l'intérêt que l'on porte à soi-même, promu au rang d'être collectif ${ }^{20}$, écrivait Jean Marcel. C'est cette promotion de l'identité collective au rang de société visible que vise Paré dans Les littérature de l'exiguité. Se situant par rapport au discours de Barthes qui, dans son Roland Barthes par lui-même, disait se tenir "derrière la porte. * coupé de la popularité du langage *, - sans cesse à l'écoute de ce dont je suis exclus", Paré réplique par une attitude d'intégration et de fraternité: "je suis à l'écoute de ce qui est exclu. (p. 62). La littérature ne saurait être *coupé[e] de ses racines communautaires et de ses appels flamboyants à l'Unité, [car] mon geste réducteur ne fait qu'illuminer les régions obscures de moi-même, de nous-mêmes, que je voulais amenuiser. (p. 61), écrit Paré, dans un glissement identitaire du • je * au *nous* (c'est Paré qui souli-

20 Jean Marcel, . Écriture et histoire -, Pensées, passions et proses, op. cit., p. 144. 
48

gne) qui donne son ton et sa couleur à l'essai, rédigé d'*une main éminemment responsable. (p. 54).

Ce glissement identitaire, cette affirmation d'un * je - qui s'accorde remarquablement à son objet, voire qui s'y subordonne, cette recherche d'une "vision du singulier-pluriel " selon la belle expression qui clôt Théories de la fragilité (p. 139), jette un éclairage sur la forme même de l'essai. En effet, l'on sera amené à établir une équivalence entre l'inscription sinueuse du "je. et l'identification de l'essayiste à la communauté de l'exiguïté, dont l'existence paraît toujours incertaine et provisoire. Il y a dans cet essai une profonde identification à la fois de l'écrivain à son objet culturel, ce qui débouche sur une valorisation du "nous " (les littératures marginales), et de la forme de l'ouvrage à son sujet: l'essai "sera ainsi lui-même l'objet d'une marginalisation, comme s'il était marqué par la contagion. (p. 9). C'est peut-être ici que l'essai, que caractérise par ailleurs la présence envahissante du * je . auctorial, fait d'abord voir la fiction qui l'engendre. L'essayiste se fait foncièrement écrivain, parce que les questions soulevées, étant celles que mettent en jeu les écrivains de l'exiguïté, conférent à l'essai sa profonde fictionnalité. De la même façon que les * petites - littératures * se sont exténuées dans le morcellement et la diversité * (p. 12), la forme de l'essai est •fragmenté[e]* (p. 11), celui-ci formant une sorte de *livre troué où ne perce pas souvent la lumière. (p. 50). Les questions abordées ne sont jamais épuisées en une seule section, elles ne le seront jamais autrement du reste; mais Paré privilégie un retour périodique sur quelques grandes questions, certains auteurs de prédilection, selon la dialectique de l'apparaître et du disparaître, typique de la marge. Le trajet de l'essayiste reproduit cette errance de l'écrivain marginalisé, qu'il s'agisse des romans de Jack Kerouac ou de ceux de Jacques Poulin, *qui ne faisaient, pour moi, que changer le mal de place, qu'ajouter de l'errance à l'errance plus ancienne. (p. 49). Tandis que le discours hégémonique travaille dans la circularité signifiante de la continuité, l'écriture de Paré, semblable au discours hétérogène, travaille dans le fragment et la discontinuité : - Les questions que j'aborde prendront tout leur sens dans la discontinuité ( (p. 30). Ainsi viennent et reviennent les questions, les auteurs commentés, parmi lesquels Patrice Desbiens qui, par son exemplarité des conditions de la littérature de l'exiguité, occupe une place de choix. Paré s'intéresse notamment à la figure de l'écrivain telle qu'elle apparaît dans L'espace qui reste: 
"morcelé[e], défait[e], un peu comme le sont les phrases déchiquetées par la structure verticale des vers" (p. 131), observe Paré, qui, en disséminant ici et là les références à Desbiens, reproduit une image du poète somme toute conforme à celle que ses recueils donnent à voir. Paré, qui traite * des mêmes lieux de la dépossession. (p. 136) que Desbiens, se trouve ainsi à construire sa fiction Desbiens ${ }^{21}$. "Comprendre une œuvre [...], c'est trouver l'angle sous lequel la perspective de l'œuvre agréera le mieux à l'époque où l'on vit", écrivait un critique français oublié, Gabriel Brunet, pour qui la critique était *une collaboration dans la création * 22 .

\section{La marque du « il »}

Pour originale que soit cette complicité formelle entre l'essayiste et son objet, elle n'aurait suffi à donner sa fiction à l'essai sans l'Autre à qui s'opposer. Le "nous " se positionnera forcément vis-à-vis d'un "il . Ce sera le discours hégémonique, celui qui aliène la parole minoritaire, qu'il condamne * aux réflexes du mimétisme * ${ }^{23}$ et qu'il empêche "d'émerger de sa secondarité congénitale (p. 36), le privant ainsi d'un accès au discours du savoir. Le pouvoir hégémonique agresse, suscite une réplique, dont la richesse tiendra d'abord au paradoxe idéologique qui la constitue.

21 Voir notamment la section - le songe de Patrice Desbiens *, titre curieux puisqu'il n'y est pas question de la relation d'un asonge. au sens propre, pas plus que de l'évocation du lieu imaginaire d'où pourrait se constituer un discours affranchi de ses marques de la marginalité. en fait, comme le laisse entendre la phrase liminaire, Paré jouc sur le signifant pour faire état précisément de cette complicité étroite qui le lie à Desbiens: * En commençànt à travailler, ce matin, j’ai encore songé à Patrice Desbiens. (p. 136; je soulignc). l.c :songe * de Desbiens, c'est dès lors l'appropriation d'une fiction sur Desbiens. - C'est pourquoi je partage avec lui cette complicité insurmontable. Du moins, c'est ce que je souhaite, ce matin, avant de me mettre au travail. (p. 137), termine Paré. C'est aussi à la lumière de Desbiens cu'il faudra lire l'ćtrange conclusion des Théurtes de la fragillté, dont le titre, * les mots qui restent *, paraphrase le titre du recucil du poète.

22 Gabriel Brunct, "Création et critique ", Mercure de France, 15 mai 1924, p. 24 25 et 29.

23 - Detrière la fragilité insoutenable et pourtant définitoire des petites littératures, s'agitent les spectres de l'altérité. Les écrivains et leurs personnages y sont particulièrement susceptibles à la métamorphose et ultimement à la perte de l'identité formelle. "rout le discours du colonisé semble intérioriser les modes de comportement du colonisateur, semble épouser les rôles du dominant; ce discours devient ainsi ke théatre d'une aliénation, d'unc prèsence en soi de l'altérité. (p. 77). 
En effet, cet essai, qui s'inscrit contre le discours hégémonique, doit pourtant s'ériger tant soit peu en un tel discours pour être entendu; il risque en somme d'être lui-même la première victime du mimétisme décrié. En s'élevant à la fonction critique, l'essayiste est menacé par l'intrusion du "il * honni dans la forme même de l'essai, et donc susceptible à son tour d'exercer le pouvoir discrétionnaire du discours dominant: "la domination habite toute la culture et est surtout identique à la fonction critique", note Paré, qui s'avoue *tourmenté par le langage de ce livre*(p.81). D'où la nécessité de repenser l'essai critique.

Toute la stratégie discursive de l'ouvrage s'expliquera, audelà de la connivence fraternelle établie par le aje ", par cette résistance critique au discours hégémonique. La profonde originalité de l'essai lui viendra avant tout par la nécessité dans laquelle se sent l'essayiste d'élaborer une forme critique qui à la fois fasse état de la précarité de l'objet culturel dont il se sent solidaire et se démarque radicalement des canons du discours théorique et critique tel qu'il s'était principalement donné à lire depuis l'emprise du formalisme sur l'essai littéraire. Il s'agit, en effet, d'assumer une fonction critique qui serait dénuée du regard en plongée qui la caractérise si bien, de refuser de se situer dans une position asymétrique en regard de l'objet, afin de préserver, précisément, l'harmonie de la magie "communale. Il s'agit, en somme, de faire le pari d'une rhétorique performative qui échappe à la rigueur du discours organisé, qui fasse tant soit peu oublier la fonction critique en montrant qu'elle est des nôtres, pour reprendre une formule de Paré 21 .

Presque naturellement, la réorganisation du discours critique, se mesurant au pouvoir hégémonique, va introduire un schème d'intelligibilité de la situation minoritaire, schème axiologique déterminé par l'opposition à l'Autre selon la structure binaire coupable/innocent. J'y insiste, car ce schème définit profondément l'indéniable fictionnalité de l'essai. Le *il " est perçu comme un ennemi, comme un agresseur qui maintient un pouvoir abusif et, qui plus est, usurpé : les littératures de l'exiguité auront été victimes de processus historiques d'exclusion institués à la Renais-

24 la formule établit le partage: François Paré reconnaîtra qu'Herménégilde Chiasson .lestl l'un des nôtres. (p. 151); en revanche, il déchantera après quelques espérances qui l'avaient amené à se - dilrel que Lyotard était des nôtres * (p. 58). 
sance européenne, celle-ci, en effet, "présidlant] à l'érection du concept de grandes littératures, issues d'un héritage littéraire, celui de l'Antiquité gréco-romaine, lui-même à moitié fictif * (p. 10). On voit, dans ces lignes capitales, fondatrices de toute l'activité critique de Paré, comment le rapport à l'Autre est posé et réfléchi, comment il sera développé : selon un rapport injuste de domination, le minoritaire ou le marginal apparaissant comme une victime qui doit sans cesse lutter pour sa survie. Aussi la volonté de l'essayiste de faire accéder la parole marginale au discours du savoir se nourrira-t-elle paradoxalement du -savoir de la résistance. (p. 5) à l'égard de l'hégémonie ${ }^{25}$. La visée cognitive du discours fera état du manque ${ }^{26}$, où il s'agira d'atteindre à la distinction par la qualité de sa différence au regard de l'Institution: " [cl'est en tant que discrimination, inscrite dans l'écriture et dans l'institution de cette écriture, que cette différence doit être aujourd'hui théorisée- (p. 155). En d'autres mots, la stratégie discursive va reposer sur une dialectique qui revendique, contre l'Autre aliénant, coupable, la différence de la victime innocente, de l'exclu, qui, de tout temps, a été privé du "privilège ontologique ; nul doute, "ce qui importe, c'est le rapport inégal au pouvoir" (p. 13).

Dans cette optique, Paré observe que * [l]e déclin de la classe intellectuelle, surtout en France, et la culpabilité qui paralyse tous les discours dominants permettent d'espérer l'émergence d'un discours de la marginalité culturelle. (p. 157). Il y aurait lieu d'évoquer, comme explication à cette situation, l'avènement d'une "culture de masse" ou encore "la civilisation de l'audiovisuel. (p. 156), peu importe; l'essentiel est que le déclin des intellectuels, dont on a assez fait état depuis quelques années, et

25 C'est cela aussi que signifie la métaphore qui ouvre l'essai et que paré reprend en conclusion : l'ćtroite bande des duncs, coriaces et placides *, qui protègent les villages de la mer, est un *exemple de résistance infinie, changeante et créative. (p. 165).

26 *Nous devons attendre des discours de l'exiguïté une réinterprétation du mancjue, de la privation de l'etre, du moins de ce que nous percevions avec des yeux autres comme une privation et un mancuc, mais qui pourrait bien être notre plus subtile richesse - (p. 157-158). Dans Théorie de la fragilité: * le minoritaire, parce qu'il est à tous points de vue minorisé, n'a pas d'origine, se caractérise par ce mancue même, qui le laisse alors fatalement condamné à une incessante dérive (p. 27). En ce qui concerne ce dernier texte, j’indiquerai dorénavant la pagination entre parenthèses après la citation. le contexte établira si je cite Les littératures ou Theories. 
la culpabilité paralysante des discours dominants (le politically correct en est la manifestation sociale la plus éclatante), sont étroitement liés, participent d'un même et seul processus historique : ils constituent la réussite incontestable du triomphe de l'individualisme hérité de la Révolution et de la Déclaration des droits de l'homme et du citoyen. L'individualisme repose foncièrement sur un refus de la responsabilité et le recours - apparemment légitime - à la satisfaction victimaire. Il semble que le discours rousseauiste, en particulier, ait constitué une étape décisive de la pensée individuelle, ou à tout le moins qu'il nous permette aujourd'hui d'expliquer la cohérence d'une évolution sociale dont son attitude rend compte de façon exemplaire. Rousseau, calomnié par les hommes et cherchant à se défendre, a pris le risque des confessions, moyen pervers s'il en est, car il signifie: je me trompe peut-être sur les événements que je rapporte, mais pas sur ce que j'ai ressenti - mon profond sentiment d'aliénation, de persécution. C'est ce qui permettra à un Pascal Bruckner, par exemple, d'affirmer que "si Jean-Jacques défriche un champ nouveau par rapport à Pascal et à Montaigne, c'est qu'avec lui l'individu naît persécuté, en proie à autrui. ${ }^{27}$. On connaît la suite: l'avènement de la société démocratique n'a cessé d'accroître son capital de fraternité et de promouvoir une (apparente) égalisation des conditions sociales à travers "l'exploitation du principe des singularités individuelles ${ }^{28}$, ouvrant ainsi la porte aux revendications de tous ceux qui aspirent au partage du pouvoir, lequel partage se donne à entendre comme un droit. La structure est pernicieuse et sa logique infaillible: le droit à la reconnaissance est conséquemment le droit de l'exclu, ou si l'on préfère, le droit d'affirmer son innocence en culpabilisant l'Autre. Enfin, l'individualisme contemporain réussit la "prouesse singulière " de présenter *comme l'ultime liberté individuelle le primat absolu de la collectivité ${ }^{29}$.

Qu'on me lise bien. Je ne dénie pas aux littératures marginales l'accès au savoir que revendique Paré, ni ne conteste la légitimité de la rhétorique victimaire, puisqu'elle participe d'un effet de société qui lui accorde d'office sa pleine légitimité. Tant il est vrai que l'es-

27 Pascal Bruckner, La tentation de l'innocence, Paris, Le livre de poche (Grasset), 1995, p. 30 .

28 Gilles Lipovetsky, L'ère du vide. Essai sur l'individualisme contemporain, Paris, Gallimard, coll. * Folio essais *, 1996, p. 14.

29 Alain rinkielkraut, La défaite de la pensée, Gallimard, coll. * Folio essais •, 1993, p. 142. 
sayiste travaille malgré lui dans l'idéologie et qu'il est traversé par l'ensemble des discours sociaux, Les littératures de l'exiguité participe d'un mouvement généralisé de découverte, voire de reconnaissance institutionnelle non seulement des littératures dites marginales, mais aussi de la paralittérature ou d'écrivains marginaux qui ont œuvré au sein d'une grande littérature, la française par exemple ${ }^{30}$. Enfin, bien sûr, je parle encore moins de la minorité au nom de laquelle l'essayiste s'exprime ou croit pouvoir s'exprimer...

On m'excusera pour cette parenthèse, mais elle est essentielle, car elle fait comprendre comment le discours de Paré crée sa propre fiction, dans quelle construction imaginaire il s'inscrit, donc à partir de quelles données socioculturelles il engendre sa fiction. Ce qui m'intéresse ici - c'est là ce que je dis -, c'est de voir comment l'essai littéraire tel que le pratique Paré "montrle] la voix de la communalité des individus dans l'imaginaire", fait "le pari essentiel de l'imaginairen, selon les termes qu'il utilise dans Théories de la fragilité (p. 135); en d'autres mots, m'intéressent la façon dont le discours construit son objet, la position du sujet énonciateur à son discours et par rapport au pouvoir, vis-à-vis duquel il recourt, de façon exemplaire, à une structure victimaire hautement performative. Au-delà du fait que la victimisation n'est en rien une fatalité, mais somme toute une tendance, et "qu'il est permis de rêver à d'autres modes d'êtres plus authentiques ${ }^{31}$, l'essayiste construit une certaine représentation de la parole marginale, à l'aide de signes linguistiques qui miment le réel, qui créent un effet de réel, lequel réel paraît couler de source vu l'investissement de l'essayiste et sa difficulté (son refus) d'abstraire l'écriture du lieu identitaire ${ }^{32}$.

La réflexion que soulève cet essai traite moins de l'Institution que de l'opposition à son égard. La différence est fondamentale,

30 Le cas le plus remarquable est certainement la réédition de bon nombre de romanciers de l'entre-deux-guerres exclus des histoires littéraires: Bove, Prévost, Beucler, Gadenne, Hyvernaud, Calet, etc. Et j'ajouterai : ces romanciers sont un peu réédités pour des considérations esthétiques et beaucoup pour cette reconnaissance de l'individualisme contemporain que leurs ceuvres illustrent. À ce propos, je renvoie à mon essai sur Bove, à parâtre à l'automne 1997 chez Nuit blanche éditeur.

31 Pascal Bruckner, La tentation de l'innocence, op. cit., p. 17.

32 Dans Théories de la fragilité: "Je ne crois pas qu'il soit possible d'écrire une ocuvre où les lieux d'appartenance identitaire ne constituent pas déjà un tracé de lecture* (p. 12). 
elle détermine la position de Paré qui s'introduit comme sujet chargé de ses expériences et sentiments, qui colore l'essai d'une subjectivité intégrale et intégrante. C'est ainsi que le travail de l'essayiste "est plus que descriptif ", qu'il devient "un combat politique contre l'impuissance. (p. 34). Fortement présent à son discours, porte-parole privilégié de la communauté marginale dont il parle, c'est par la revendication trouble du savoir de la résistance que Paré cherche l'accès au discours du savoir; le discours fait d'abord acte de sa propre capacité à se situer vis-à-vis de l'Autre, qu'il investit de présupposés critiques qui s'approprient et redistribuent la figure de l'Autre selon un schème d'intelligibilité particulièrement efficace et propre à se faire entendre en cette fin de siècle individualiste. Il y eut vraisemblablement, dans le processus historique des formes canoniques et génésiques des grandes littératures, un déni des petites cultures, "bannie[s] par définition de l'Histoire mémoriale* (p. 40), mais surtout ce processus, dans les essais, est intimement relayé par l'héroïsme de la marge, par la nécessité d'une "rédemption de la communauté par l'écriture " (p. 80), qui surdétermine un tel rapport conflictuel. La littérature marginale apparaîtrait peut-être moins déniée par un développement historique qu'elle n'alimente cette dénégation au profit de la sacralisation de sa propre exclusion, rédemptrice et transfigurante, qui devient le seul mode d'affirmation possible, de revendication privilégié, tant il est vrai que, "pour qu'un discours ait du pouvoir, il doit mettre en marche une croyance. 33 . Pour reprendre une expression récurrente du discours de Paré, qui exprime bien le mimétisme de l'écriture dans sa prétention à reproduire le réel, et qui traduit tout à fait l'habilité de ce discours à jouer de l'ambiguîté qu'il cultive, tout se passe comme si ${ }^{31}$ l'exiguité et la fragilité triomphaient de, mais à même, l'altérité aliénante qui les constitue. * Les littératures de l'exiguïté n'ont, pour richesse ultime, que le mot peut-être. (p. 166), écrit Paré

33 Paré (p. 47) cite ici Elisco Vernon, "Semiosis de l'idéologie et du pouvoir*, dans Communication, $\mathrm{n}^{\circ} 28$ (1978), p. 16. Le discours est conforme à l'attitude poétique de la contre-culture qui * a dû, pour établir les conditions propres de son intervention dans le monde, inventer de toutes pièces, dans un temps et un espace immémoriaux, son mutisme et son exclusion de la parole* (p. 104 ; je"souligne).

34 Un seul exemple, mais particulièrement signifiant: *Tout se passe dès iors comme si les conditions de marginalisation étaient devenues, à travers leur appropriation par le discours littéraire dominant, les faces privilégiées de ce discours " (p. 20). 
pour clore son essai. Le mot fait état de l'espoir dans la reconnaissance possible de la marge, mais je ne peux m'empêcher de lire, dans cet italique, tout l'artifice d'un discours qui construit précisément ce qui peut être.

\section{Le mythe Paiement}

Il y a donc tout un art de la feinte chez Paré, un art subtil de la mise en scène qui converge vers la représentation (théâtrale) du «je . héroïque et de la communauté humiliée. Cela se donne à voir de façon particulièrement éloquente dans Théories de la fragilité, où l'essayiste s'engage à "produire l'illusion de la communalité - (p. 12), car tout $*$ n'la] de sens que dans la reconnaissance entêtée du rassemblement. (p. 139). Je voudrais m'attarder quelque peu à cet essai, avant de saisir l'ensemble de la dialectique pronominale en fonction de la question du Père. Il y a au centre des Théories un héros, ou plus exactement un anti-héros exemplaire: André Paiement, l'auteur des premières pièces du Théâtre du Nouvel-Ontario, chez qui *les êtres ne trouvent le réconfort que dans les limites de la communalité (p. 76). Paiement, qui s'est suicidé à vingt-huit ans, incarne, depuis l'article de Fernand Dorais ${ }^{35}$, la figure emblématique de l'écrivain sacrifié par les conditions aliénantes de la marginalité sociale. Dorais récupère l'événement par le mythe. Dans le chapitre qu'il consacre à Dorais, Paré y étudie .l'élaboration d'une théorie de la minorisation" vue "comme production artistique vivante. (p. 35), qui fait état d'*une revendication triomphante* (p. 34) et se sert de Paiement comme "métaphore de l'existence collective et [...] confirmation de notre libération éventuelle par le représentation discursive" (p. 32). Le discours de Dorais, qui comporte sa bonne part de "mystification" (p. 32), participe d'aun certain lyrisme de la dépossession* (p. 29). Paré note encore: "Cet épisode sudburois, dont le grand prêtre sacrifié dans la personne d'André Paiement est la caution sacrale, devient notre origine, jusque-là manquante, surtout le lieu d'origine du littéraire dans notre société si privée d'images d'elle-même (p. 31). Nous sommes bien au théâtre, rien n'y manque, et c'est là en fait *l'épisode*, le

35 * Mais qui a tué André?*, Revue du Nouvel-Ontario, no 1, 1978, p. 34-57; article repris dans Entre Montréal... et Sudbury, Sudbury, Prise de parole, 1984, p. 15-33. 
chapitre central d'un drame collectif qui rejoue Lavalléville en postulant la consécration d'un mythe qui *annonce [...] une rédemption collective. (p. 75), selon les mots de Paré commentant la pièce de Paiement. Bref, le discours de Dorais est une "invitation à la Fête. (p. 29), de la même façon d'ailleurs qu'il semble à Paré que, chez Paiement, - les minorisés sont tout fin prêts à basculer, tête première, dans la fête * (p. 77).

Le discours de Paré s'inscrit exactement dans cette mythologie élaborée par Dorais, à qui est significativement consacré le premier chapitre d'analyse des Théories de la fragilité. Dorais reprend là où Paiement s'était arrêté, comme Paré relaiera leurs voix en s'inscrivant de plain-pied dans cette fiction, en filant la métaphore de la fête sudburoise ${ }^{36}$ qui sous-tend la volonté critique. Théorie de la fragilité s'ouvre sur un exergue de Robert Dickson, qui écrivait dans Abris nocturnes: *Salut, salut, salut... On m'a donné ce cahier le $1^{\text {er }}$ février $1978^{37}$, à l'aréna de Hanmer [...]. C'était un cahier à André Paiement. Il n'en avait plus besoin, vu les circonstances immédiates ( $(p .7)$. Le cahier de Paiement, passé entre les mains de Dickson, projette maintenant son ombre sur celui de Paré: -Ainsi, le cahier orphelin de l'écrivain est passé de main en main dans un geste garant de l'éternité. Le travail consterné, temporairement arrêté, figé de stupeur pouvait alors reprendre. Heureusement. [...] Le mien, mon cahier, est un peu trop grand, comme cette tablette [...] que je garde dans

36 La libération passe par le Nord pour Dorais et la contre-culture franco-ontarienne, qui reprennent la symbolique québécoise des années soixante (lacjuelle par aillcurs revient en force dans le roman québécois des années 90). Che\% Cano-musique, par exemple, les titres des deux premiers albums se lisent dans une continuité symbolique marquée par l'espoir dans le Nord. in effet, le titre Tous clans l'même bateau articule une métaphore maritimo de la fraternité communautaire de la coopérative qui prendra sens dans le cadre d'une action qui s'engage *à la poursuite du Nord", d'une identité culturelle qui *Idlébouche comme un grand fleuve au large., selon les mots de Paiement dans * En mouvement*, seconde partie de la chanson "À la poursuite du Nord. de l'album $A u$ nord de notre vie. Ainsi, nul hasard si Paré, dans l'incipit des Littératures, écrit devant la mer du Nord, - qui résume toute la sacralité de cette quête de l'espace - (p. 164) que l'essayiste cherche dans la " reconquête l...l des espaces discursifs - (p. 163). Posture symbolique qui introduit Théories de la fragilité, où la réflexion sur Dorais et Paiement "constitue le centre stratégique de ce livre. Car le Nord rejeté, il ne cesse néanmoins de se reproduire, figure du jardin génésique, comme une image un peu délavée représentant dans notre commencement même (p. 18). André Paiement est décédé en janvier 1978. 
un quelconque classeur pour le jour où j'écrirai le livre, celui qui attend. (p. 7-8). Ce nouvel incipit est fondateur, c'est le cas de le dire, du geste théâtral et sacral de l'écriture, du discours critique, de la représentation collective à laquelle souscrivent èn s'y fondant ses principaux acteurs." Il y a là en effet une sorte de passation, non pas des pouvoirs (le mot serait assez mal venu), mais disons de la parole qui fait acte de résistance, qui relève du rituel et du sacral; une transmission de la parole dans *le partage des mots* (p. 7) qui tient presque du pacte inviolable, et qui prescrit une attitude critique précise, adaptée aux conditions socioculturelles dont participe l'écrivain: une attitude qui place le *je», plus encore que dans Les littératures de l'exiguitté, au service d'un "nous" englobant et bienfaisant, autrement dit qui convoque le discours théorique à consentir à la "fête ", au détriment de la position de domination qui lui est en principe inhérente. "Quant à la signification du mot théorie, on sait qu'il s'agit aussi d'une ambassade, d'une procession, d'une fête* (p. 9), écrivait Philippe Sollers dans Théories des exceptions, que Paré met en exergue au chapitre second, où, après avoir précédemment élevé le "nous * comme phare à la dramaturgie de l'écriture, il exposera sa conception critique.

Cette fiction Paiement participe donc étroitement à la définition de la pratique critique de Paré, qui disqualifie le regard critique et fait corps avec la communauté. Dans un article de 1989, Paré considérait déjà le * mythe "de Paiement, *qui allait inspirer toute une génération récente d'écrivains et d'artistes de l'Ontario français . 38 , à la lumière de la défiance du milieu franco-ontarien à l'égard de la fonction critique, qui : semble imposer son langage, comme le fait l'Autre absolu, [et] conduit à une terrible impression d'être dépossédé de l'être communautaire propre "39. - Voilà que ce mythe, qui est évidemment une belle mystification, ne se laisse nullement analyser et résiste ainsi à la lucidité critique ${ }^{40}$, écrivait-il. C'est bien dans cette optique qu'il faut lire Théories de la fragilité, dont le titre exprime on ne peut mieux l'ambivalence structurelle de l'essai entre la fonction critique et la collectivité minoritaire. L'essai s'énoncera travail sur le fragile,

38 François Paré, * S'inscrire dans le champ du savoir *, Atmaspbènes, $\mathrm{n}^{\circ} 3,1989$, p. 18.

39 Ibid., p. 17

40 Ibid., p. 18. 
mais plus encore peut-être travail $d u$ fragile. Le mot * théorie * traduit la volonté de l'essayiste d'inscrire la précarité de la littérature franco-ontarienne dans le champ du savoir; mais comment aussi ne pas lire que la théorie est fragile, qu'elle ne saurait, en raison même de la précarité de l'objet dont elle traite, s'instituer pleinement en discours théorique? Autrement dit, le discours approchera le plus possible d'un idéal théorique constitué d'un "je" pleinement assumé mais qui ne saurait se soustraire aux conditions "fragiles * dont il témoignera, d'un idéal discursif où la théorie est à la mesure de la fragilité du moi, concommitante à son expression tourmentée entre le savoir et le vécu intuitif, entre l'universitaire et le social. *Voici donc que je me remets à élaborer des théories, "plus frêles que les pyramides stables". C'est qu'il y a des jours où la joie de la fragilité est d'une intensité fondatrice; je voudrais alors reposer comme une fine poussière sur les rivages ouverts du livre, disparaître à jamais dans ce secret voilement de la transparence qu'est l'écriture. (p. 10). Au-delà de l'analyse textuelle, le * je * ne se manifestera pas sans solidarité à la fois politique et ontologique, pourrait-on dire, avec l'Autre; idéalement, le *je. s'abolira dans un accord communal, à tout le moins jettera quelques lueurs qui parleront "de sa présence orpheline ${ }^{41}$ (p. 10). Telle sera la ligne de conduite du discours des Théories de la fragilité, nécessairement acquis à "l'excessif", où le sujet énonciateur "constituer[a] la preuve irréfutable de l'excès ${ }^{42}$ (p. 12), un peu comme Dorais avait pêché par * une certaine exagération. (p. 29).

41 Référence ultime à Paiement, *mon prédécesseur * (p. 7), écrit Paré. Dans le chapitre qu'il lui consacre, il écrit: "Bienheureux celui qui a pu faire corps avec sa communauté au point de s'y voir disparaitre totalement!* (p. 76), paraphrasant la formule liturgique.

42 Ia position de Paré est assez compatible avec celle d'André Brochu: - la prise du critique sur l'œeuvre, même quand elle s'accomplit par des clétails bien circonscrits, a toujours quelque chose de totalitaire et, d'une certaine façon, de démesuré, car la critique vise l'œuvre comme infini du sens, à travers les modes finis de l'écriture. Il n'y a pas de critique, c'est du moins une hypothèse qu'on peut s'amuser à̀ envisager, sans une mystique de l'couvre, qui fait d'elle un lieu de réunion et de communion, une subjectivité partagée et cependant transcendante ( La visée critique, op. cit., p. 142). La principale différence tient à la méfiance de Paré à l'égard de la saisie *totalitaire - qui, chez Brochu, nuance la .communion. On notera aussi que cette position s'inspire à l'évidence de la crítique de Starobinski, chez qui nous retrouvons la pensée en mouvement, le concept de la critique comme fête et le souci, comme le notait Paré lui-même en citant L'oeil vivant, It: La relation critique, 


\section{La fiction du Père}

Jamais, nulle part, en termes de psycho-sociologie, la Figure du Père ne se sera dressée si écrasante [...].

- Fernand Dorais ${ }^{13}$

Je parlerais volontiers d'une fiction du Père pour caractériser, dans son ensemble, les essais de Paré, puisque c'est bien de cela qu'il s'agit ici, du moins dans la mesure où toute production textuelle transige avec des valeurs symboliques. L'essayiste qui, dans Les littératures de l'exiguité, conteste la forme consacrée de l'essai au profit de nouvelles règles d'écriture, pour qui les chapitres, "dans leur déploiement agressif, présomptueux * (p. 30), renvoient à une structure du pouvoir critique qui agit *comme une censure intolérable (p. 58), place indéniablement son entreprise scripturaire sous le signe d'une relation conflictuelle à la figure paternelle. Paré s'inscrit contre l'Ordre intimé du langage, déconstruit, défigure, remodèle. Robert Major avait considéré comme des * justifications spécieuses * les revendications de Paré d'un discours qui refuse la construction rigoureuse de chapitres et un discours bien structuré; a et d'autant plus que le discours de François Paré est effectivement construit, sinon en chapitres, du moins autour d'un certain nombre de constellations thématiques, et qu'il est un salutaire discours critique, assez structuré 44 . J'ai moimême signalé qu'il fallait se méfier de l'allure improvisée du discours. Mais dans cette insistance de Paré à l'égard des * structures du discours critiques. (p. 58), je veux moins y voir des justifications spécieuses qu'une volonté symptomatique de suspicion à l'égard du pouvoir et une intention de profonde remise en question, voire de destruction. Que les revendications de Paré tendent à excéder en intention la forme de son discours, il me semble que cela doit compter, que cela doit être entendu et considéré à sa pleine mesure. Avant de faire profession d' a anti-intellectualisme ", selon le mot de Major, l'essayiste récuse la Loi du Père;

"cu'il n'y ait plus seulement acquiescement à l'ocuvre littéraire, mais rencontre avec celle-ci * (-S'inscrire dans le champ du savoir *, op cit., p. 12).

43 À propos de la communauté franco-ontarienne, dans * Mais qui a tué André?", Entre Montréal... et Sudbury. Prë-textes pour une francopbonie ontarienne, op. cit., p. 26.

44 Robert Major, * De la marge et des marginaux -, dans Voix et images, vol. XVIII, $\mathrm{n}^{\circ} 3$, printemps 1993 , p. 582. 
"[r]aconter, n'est-ce pas toujours chercher son origine, dire ses démêlés avec la Loi?: 45 se demandait Barthes.

À cet égard, on tiendra pour décisif le passage suivant, le seul où Paré aborde la question du Père, et encore avec une certaine prudence (pudeur ${ }^{46}$ ), préférant assigner l'évidence que développer l'idée: *En fait, toute écriture dans les conditions de minorité (et au fond toute la littérature émane de telles conditions!) est une remise en question de la figure paternelle, religieuse ou non* (p. 100). Il me semble qu'on doive lire l'essai à la lumière de cette assertion, qui nous conduit directement sur les traces de l'écriture narrative; elle explique l'imposante intensité du "je* et cette sourde revendication, inscrite dans la marge (c'est le cas de le dire) du texte et sa visée dans le champ du savoir, d'un exceptionnel statut d'écrivain à part entière. En inversant la Loi, l'écrivain aspire lui-même à la position paternelle. Il tente ainsi une incursion en territoire paternel, il renverse l'interdit et revendique le plein droit à la posture du Père. « Nul objet n'est dans un rapport constant avec le plaisir. [...] Cependant, pour l'écrivain, cet objet existe; ce n'est pas le langage, c'est la langue, la langue maternelle. L'écrivain est quelqu'un qui joue avec le

45 Roland Barthes, Le plaisir du texte, Paris, Seuil, coll. - Points", 1982, p. 75. Sous cet angle, on envisagera aussi l'écriture de Desbiens, à qui j'ai fait référence plus haut, et dont Flizabeth Lasserre a mis en évidence une attitude littéraire qui s'énonce comme une "contestation générique *, une · contestation de la notion d'écrit - et une *contestation du pouvoir de la langue. " Un poète au seuil de l'écriture: l'exiguïté selon Patrice Desbiens ", dans Lucie Hotte et François Ouellet (dir.), La littérature franco-ontarienne: enjeux esthétique, op.cit., p. 27-42). Paré a indiqué que la lecture de Desbiens a constitué - Isla toute première source de questionnement. À partir d'elle, je ne pouvais plus voir la littérature, dans ses miroitements hégémoniques, comme je l'avais toujours vue: solide, belle et consolante à l'extrême. Tout avait changé. Pour le mieux? Je n'en sais encore rien, à vrai dire. Patrice Desbiens en veut mortellement à l'institution littéraire (et universitaire surtout), qu'il juge profondément aliénante et carrément méprisante- (p. 136).

46 Témoin cet autre passage où $u$ aurait pu être question du père - mais néanmoins coiffé d'un titre significatif: * Fracasser les carreaux de la maison du Père. Il se lit intégralement comme suit: "Non, je ne parlerai pas du livie merveilleux de Patricia Smart, auquel j'aurais pu emprunter nombre d'exemples et un peu de cette rare intelligence de l'oppression véritable. Je vous renvoie plutôt à la lecture obligatoire de ce livre-(p. 118). En réalité, l'essai débat obstinément de la figure du Père en lui substituant : un pouvoir injuste et illégitime (p. 33). - On demande à l'écrivain de faire comme si son travail n'avait rien à voir avec le pouvoir et l'institution du pouvoir. Rien n'est moins vrai - (p. 32). 
corps de sa mère [...] pour le dépecer, le porter à la limite de ce qui, du corps, peut être reconnu: j'irai jusqu'à jouir d'une défiguration de la langue, et l'opinion poussera les hauts cris, car elle ne veut pas qu'on "défigure la nature" ${ }^{47}$. C'est ici, dans un sens, que l'essai balance définitivement de tout son poids dans la fiction: l'affirmation centrale du *je , supportée par un rappel constant de l'impuissance et de l'angoisse de la marge communautaire (performance discursive d'ordre essentiellement ontologique), est constitutive de la mobilisation d'un imaginaire agressif qui réforme la langue. "Je veux mettre en scène la disparition de l'Un, dont j'ai toujours eu grande crainte. (p. 158). Le sujet énonciateur est foncièrement narrateur, il participe d'un schéma narratif à trois acteurs: le *je*, figure de fils, vis-à-vis d'un *il *, figure de Père; entre ces identités pronominales, un "nous * (figure communautaire) dont le * je * est partie prenante, mais à qui la caution du «il * est nécessaire pour accéder à la dignité littéraire, pour s'inscrire dans le champ du savoir.

Ce schéma est exactement conforme au roman franco-ontarien tel qu'il se présente chez Daniel Poliquin, par exemple, en particulier dans L'écureuil noir, roman de l'écriture, de l'élaboration de la possibilité d'un discours littéraire vis-à-vis de l'autorité. Je rappelle brièvement comment la relation au père est posée dans L'écureuil noir ${ }^{48}$. Le roman s'ouvre sur la décision du héros, Calvin Winter, de changer d'identité, de devenir un autre homme, décision prise après avoir veillé son père, condamné, qu'il était chargé de débrancher. Calvin, profondément marginal, qui se définissait comme "la victime parfaite " ${ }^{49}$, n'a jamais su s'intégrer dans la société. Or, cet état de chose changera graduellement à la suite du meurtre symbolique du père par Calvin, qui réhabilitera dans sa conscience la figure paternelle en redéfinissant son rôle et sa fonction: la relation idéale au père doit substituer au rapport dominant/dominé un rapport d'égalité, condition même de l'intégration sociale du fils. Enfin, Calvin est aussi écrivain. Il a autrefois travaillé comme nègre, mais maintenant qu'il a résolu le conflit qui le liait au père, qui aliénait sa liberté, il peut écrire, il

47 Roland Barthes, Le plaisir du texte, op. cit., p. 60.

48 Pour une étude plus approfondie de cette relation, je renvoie le lecteur à mon article "Se faire père. L'ouvre de Daniel Poliquin -, dans Lucie Hotte et François Ouellet (dir.), La littérature franco-ontarienne: enjeux estbétigues, op. cit., p. 91-116.

49) Daniel Poliquin, L'écureuil noir, Montréal, Borćal, 1994, p. 11. 
sait écrire, produisant ce récit qu'est L'écureuil noir, dont la légende métaphorise son propre destin ${ }^{50}$. L'écriture est le témoignage, la trace concrète, d'une rédéfinition identitaire vis-à-vis du pouvoir.

On reconnaît là assez facilement la structure idéologique des Littératures de l'exiguité (car il faut bien reconnaître que si "l'essai verse dans la fiction ", en revanche "[l]e roman est mangé par l'essai ${ }^{51}$ ). La relation à l'autre n'est pas un but en soi, mais bien le moyen par lequel il est permis au aje. d'espérer établir un rapport équitable à la réalité, à la société. Comme chez Poliquin, il s'agit pour Paré de redéfinir, par l'écriture, la relation au Père, gage de la réorganisation symbolique d'une Institution dont le pouvoir hégémonique est contesté. C'est à cette condition seulement que la communauté marginale pourra trouver sa place au sein d'une structure sociale d'ensemble, mouvement de reconnaissance auquel Les littératures de l'exiguité contribuerait en posant un premier jalon critique ${ }^{52}$. Il s'agira donc de concevoir un nouveau rapport au pouvoir, la mise en place d'un rapport d'égalité dans un cadre de distribution de la parole: "la recherche d'un langage hégémonique, fondé dans l'unité de l'Être, par la "communalité " des différences fondée dans la pluralité de l'être " (p. 162), écrit Paré.

Pourtant, en dépit des ressemblances, Paré, dans son analyse sur la figure de l'écrivain dans les romans de Poliquin, n'aura pas dégagé la structure même qui oriente sa propre démarche signe indéniable de l'ambiguité et de la complexite de la posture scripturaire endossée. En fait, la découverte qu'il fait, chez Poliquin, de l'articulation d'une structure narrative "qui tend à

50 - Ces écurcuils noirs seraient en réalité d'anciens rats qui se seraient mêlés à des écureuils gris pour éviter les mesures de dératisation. [...] Ce matin-là, j’ai eu envie de disparaître comme eux dans le décor, de me rendre méconnaissable et sympathique. Changer de peau, me réinventer, mais en mieux . (lbid., p. 17).

51 André Belleau, , Petite essayistique •, Surprendre les woix, op. cit., p. 86.

52 Contribution assez réussie compte tenu de la réception critique de l'essai et des distinctions qu'il aura obtenues. Cela m'amène à cette interrogation : estce un hasard si L'écureutl notr, qui recevra un accueil critique unanimement élogieux, sera lui-même distingué par le prix du Gouverneur général 1994 (catégorie roman), ainsi que par le prix littéraire 1995 du journal Le Droit? Je pourrais poursuivre: est-ce un hasard si la pièce de Michel Ouellette, French Town, qui met en scène une relation père et fils dès plus problématiques, a elle aussi reçu le prix du Gouverneur général 1994 (catégorie théâtre)? 
réduire au maximum la présence autoritaire de l'écrivain et à dénoncer la décadence générale des institutions. .53 - assertion éminemment constestable par sa simplification ${ }^{54}$ - participe de la construction imaginaire de la marge. C'est en regard de la contestation d'une autorité paternelle inique, que vise une réforme de la pratique littéraire, qu'il faudrait considérer l'insistance de Paré à nier, au profit des figures de l'oralité 55 , la * figure honteuse. (p. 123) de l'écrivain dans le corpus franco-ontarien.

Enfin, tant chez Poliquin d'ailleurs que chez Paré, la manifestation extrême du "je ", la surenchère de la forme "communale *, le recours au schème victimaire et l'opposition au pouvoir - chacun de ces aspects, liés les uns aux autres, contribuant à créer la littérarité de l'essai —, s'expriment par la valorisation du champ sémantique du religieux, et actualisent la fonction littéraire dans sa relation sacrale à la communauté opprimée. Chez Paré, l'interrogation suivante trahit assez bien la présence diffuse du religieux dans le discours: "On m'amène encore malgré moi (de quoi ai-je le contrôle, à bien y penser?) à parler de religion. (p. 46). En réalité, Paré n'a jamais directement abordé la question religieuse, et c'est ce dont rend compte la tournure indirecte de sa phrase, qui signifie que, en somme, de religion il est constamment question par la couleur et l'ambiance du discours, par la figure sacrale du Père qui couve sous la question. La forme interrogative, la

53 François Paré, " La figure du disparu. Daniel Poliquin •, Théontes de la fragilité, op. cit., p. 113.

54 Voir à ce sujet les pages 107-110 de mon article :Se faire père. L'œuvre de Daniel Poliquin *, op. cit.

55 Bien qu'il soit vrai que la littérature franco-ontarienne (comme d'autres littératures marginales) entretienne une certaine méfiance à l'égard de l'écrivain, préférant nourrir le socle identitaire de soirées de poésie et de chanson ou encore de représentations théâtrales, : le récitatif par excellence - - au -sein des littératures de l'exiguité- (p. 107), écrit Paré dans Les littératures. En ce qui concerne l'Ontario français, les premières manifestations slittéraires - ne sont pas romanesques, pas même scripturaires: le point de départ de ce dont on s'autorise aujourd'hui pour parler de *la littérature franco-ontarienne -, c'est la création du Théâtre du Nouvel-Ontario en 1971 et les Nuits sur l'étang (à partir de 1973). C'est en 1973 aussi que seront fondées à Sudbury les éditions Prise de parole, dont le nom est on ne peut plus significatif: il s'agit certainement d'une volonté d'accéder à -l'âge de la parole -, mais d'une certaine manière, qui tend précisément à exclure l'écrit (ce n'est qu'à partir de 1985 que la maison d'édition décidera d'accorder une priorité au roman). Sur ces questions, voir Gaston Tremblay, Prendre la parole. Le journal de bord du grand CANO, Ottawa, Le Nordir, 1996. 
neutralité du sujet *on * qui éclipse un * malgré moi * qui s'excuse presque de penser tout haut, laisse voir une fois de plus le tabou qui entoure la question, ou plutôt combien cette question oriente secrètement tout le discours. L'on a vu aussi, a propos du discours de Paré sur Dorais et Paiement, combien les Théories de la fragilité développait une isotopie du sacré. Il est vrai que le plan de l'ouvrage, en regard de celui des Littératures de l'exiguité, est marqué par une rigueur beaucoup plus soutenue dans l'agencement thématique ${ }^{56}$, ce qui dénote l'intention plus nette d'un discours critique qui permettrait de faire accéder, même dans une faible mesure, la littérature franco-ontarienne au rang des discours du savoir; mais ce souci de construction semble avoir eu pour effet de produire, en réaction, une valorisation compensatoire "excessive - de la forme "communale " et de sa dimension sacrale, une force de conviction comparable à celle de Dorais qui faisait verser son œuvre "dans le discours sacré " 57 (p. 35).

- Y a-t-il une certaine grandeur à tirer de l'étude du microscopique?. (p. 9) se demandait François Paré au début des Littératures de l'exiguité. La réponse ne fait aucun doute, et la question,

56 L'essai est constitué de seize chapitres. Onze sont consacrés à un ou des écrivain(s), où la présence de l'instance énonciatrice s'impose moins que dans Les littératures de l'exiguité; en revanche, le *je. donne sa pleine mesure dans les cinq autres chapitres, où l'essayiste fait état de conditions théoriques et critiques qui définissent les littératures marginales, la francoontarienne principalement.

57 C'est tout le rapport de la critique à la communauté en regard du savoir qu'interroge Paré dans ses demiers articles. Dans * Pour rompre le discours fondateur: la littérature et la détresse - (dans La littérature franco-ontarienne: enjeux estbétique, op. cit.), Paré, craignant une sclérose d'un discours critique qui serait incapable de se situer en dehors des - grandes lignes de la pensée de la communauté tout entière. (p. 12), souhaite poursuivre une pratiçuc critique qui engendrerait a des conditions de rupture et de transformation, à la fois dans le tissu culturel et dans Isla façon singulière de le concevoir. (p. 14). L'essayiste se place en accord avec la nouvelle littérature francoontarienne qui articule "la fragmentation difficile, douloureuse, du récit fondateur, seule pourtant capable de fonder l'accession de la culture minoriscé au champ du savoir- (p. 24). Paré reprend ce discours dans " La chatte et la toupie: écriture féminine et communauté en Acadie. (dans Francopbonics d'Amérique, $\mathrm{n}^{\circ} 7$ [19971). Paré observe l'émergence, dans les années (juatrevingts, d'une modernité acadienne qui remet en cause - toute une pensée commune de l'origine et du destin des individus [...], de même que la fonction sacralisante de l'écrivain en tant que porte-parole prophétique de sa collectivité. (p. 119). - Le savoir découle de la rupture, de la désinvestiture du passé - (p. 124), conclut-il. 
du reste, relève de la rhétorique, elle vise à orienter l'angle de lecture, elle prépare déjà le lecteur à être attentif à la façon dont l'écriture opérera, au profit de la marge héroïque, un renversement de sa situation d'écriture à elle-même pour instruire graduellement un procès aux formes hégémoniques dont il importait de "circonscrire les processus d'exclusion" (p. 11), générateurs d'une pensée régionalisante. C'est là la vertu du signifiant, qui invente le signifié, transforme en imposant son regard sur le réel, se transforme pour se donner pour le réel lui-même. *Et l'essai, en ce sens, appartient peut-être autant à l'ordre du poème qu'à celui du discours . ${ }^{58}$, écrivait fort justement François Ricard. Qu'est-ce à dire, sinon, comme le disait Valéry, que "[l]'écrivain véritable est un homme qui ne trouve pas ses mots. Alors il les cherche. Et en les cherchant il trouve mieux "59. L'essayiste est aussi cet écrivain, il cherche, il trouve, il construit, il élabore; surtout il découvre, et découvrant il se découvre. C'est-à-dire qu'il ne sait pas exactement ce qu'il trouvera, mais il sait qu'il fera une découverte, et cette découverte l'émerveille, le restitue au monde dont il s'était momentanément écarté. Il s'agit pour l'essayiste, pour le critique littéraire qui peut se dire essayiste, * d'imaginer le vie même ${ }^{(x)}$. Et, sans doute, pour cette raison, ce n'est pas tant la vie qui l'intéresse que les significations qu'il peut en tirer, le sens qu'il peut lui donner. C'est lui qui aura le dernier mot: - S'agit-il d'une illusion? Sans aucun doute, mais cela n'a aucune importance. Nous sommes toujours dans le domaine du représenté. (p. 32), lit-on dans Théories de la fragilité.

58 François Ricard, La littérature québécoise contemporainc. 1960-1977 Il'essail •, Études françaises, vol. XIII, nos 3-4, octobre 1977, p. 367.

59 Paul Valéry, - Poïéticue *, Cabiers, t. II, Gallimard, coll. - Bibliothèque de la Pléiade., 1980, p. 987.

6) Michel Butor, "La critique et l'invention *, Répertoires III, Paris, Minuit, 1968 , p. 14 . 